Gut and Liver, Vol. 9, No. 6, November 2015, pp. 714-719

\title{
Treatment Strategy after Endoscopic Resection of Superficial Esophageal Squamous Cell Carcinoma: A Single Institution Experience
}

\author{
Hyun Jik Lee, Hyuk Lee, Jun Chul Park, Sung Kwan Shin, Sang Kil Lee, and Yong Chan Lee \\ Department of Internal Medicine, Institute of Gastroenterology, Yonsei University College of Medicine, Seoul, Korea
}

See editorial on page 693.

Background/Aims: The aim of this study was to analyze and propose a treatment strategy after endoscopic resection of superficial esophageal squamous cell carcinoma in a single institution. Methods: This is a retrospective review of 37 patients who were treated by endoscopic resection during a 6-year period. Results: The mean tumor size was $11.5 \pm 5.5$ $\mathrm{mm}$ (range, 3 to $31 \mathrm{~mm}$ ). Thirty-one lesions (83.8\%) were treated by endoscopic submucosal dissection, and six lesions were treated by endoscopic mucosal resection (16.2\%). The en bloc resection rate and complete resection rate were $91.9 \%$ and $81.8 \%$, respectively. The tumor invasion depth was diagnosed as epithelial in five cases (13.5\%), lamina propria mucosa in 12 cases (32.4\%), muscularis mucosa in 10 cases $(27.0 \%)$ and submucosa in 10 cases $(27.0 \%)$. The complication rate was $13.5 \%$ and included three cases (8.1\%) of perforation. Ten patients who had muscularis mucosa and submucosa lesions received additional treatments, including six patients who were treated with esophagectomy, three patients who were treated with radiotherapy and one patient who was treated with chemoradiotherapy. One patient with lamina propria lesions received radiotherapy due to a positive resection margin. The median follow-up duration was 22 months (range, 4 to 79 months), and no recurrence or metastasis was noted during follow-up. Conclusions: Tailored management after endoscopic treatment of superficial esophageal squamous cell carcinoma can offer an acceptable oncologic outcome in early esophageal carcinoma. (Gut Liver 2015;9:714-719)

Key Words: Endoscopic resection; Treatment strategy; Superficial esophageal squamous cell carcinoma

\section{INTRODUCTION}

Esophageal cancer represent the eighth most common malignancy and the sixth leading cause of cancer mortality. ${ }^{1}$ In South Korea, esophageal cancer is currently eighth leading cause of cancer death. ${ }^{2}$ Health check-up has recently become more popular for screening purposes and esophagogastroduodenoscopy (EGD) is performed biannually in individuals older than 40 years by Korea's National Health Insurance check-up program due to the high incidence of gastric cancer. So the incidence and detection rates of superficial esophageal squamous cell carcinoma (ESCC) are seemingly increasing.

Recently endoscopic resection (ER) is recognized as a possible curative treatment option for ESCC confined within the mucosal epithelium or lamina propria mucosa. ${ }^{3-6}$ Although esophagectomy has been the standard therapy for superficial ESCC, ER has gained widespread attention in treatment option. In ESCC with epithelium or lamina propria mucosa invasion, the risk of lymph node is minimal. In contrast, when tumor invades muscularis mucosa or submucosa, the risk is raised up to $10 \%$ to $40 \% .^{7-9}$ However, there are still limitations in accurate diagnosis of the lymph node metastasis before performing ER. And little information is available regarding comparative efficacy of endoscopic treatment in superficial esophageal cancer, and optimal management plan for these patients remains unknown. The aim of this study was to evaluate the efficacy, complication and long-term outcome of ER, and propose a treatment strategy after ER of superficial ESCC in a single institution experience.

\section{MATERIALS AND METHODS}

\section{Patients}

From May 2007 to August 2013, 37 patients with superficial ESCC were treated by ER at Severance Hospital, Yonsei Uni-

Correspondence to: Yong Chan Lee

Department of Internal Medicine, Yonsei University College of Medicine, 50 Yonsei-ro, Seodaemun-gu, Seoul 03722, Korea

Tel: +82-2-2228-1960, Fax: +82-2-393-68840, E-mail: leeyc@yuhs.ac

Received on April 14, 2014. Revised on July 3, 2014. Accepted on July 7, 2014. Published online December 5, 2014

pISSN 1976-2283 eISSN 2005-1212 http://dx.doi.org/10.5009/gnl14142

() This is an Open Access article distributed under the terms of the Creative Commons Attribution Non-Commercial License (http://creativecommons.org/licenses/by-nc/4.0) which permits unrestricted non-commercial use, distribution, and reproduction in any medium, provided the original work is properly cited. 
versity College of Medicine. Inclusion criteria were newly diagnosed ESCC confined to the mucosa or submucosa invasion, absence of clinical evidence of lymph node and distant organ metastasis with no previous treatment history. All visible lesions were classified according to the Japanese classification..$^{10}$ The superficial ESCC was categorized as subtype 0 and subclassified as below. Type 0-I, superficial and protruding type; Type 0-II, superficial and flat type; Type 0-IIa, slightly elevated type; Type 0-IIb, flat type; Type 0-IIc, slightly depressed type; Type 0-III, superficial and excavated type.

Before ER, endoscopic examination with lugol solution chromoendoscopy, and computed tomography (CT) of chest and abdomen or positron emission tomography-computed tomography (PET-CT) were performed in all the patients. Endoscopic ultrasonography (EUS) was examined in most of patients to evaluate depth of cancer invasion and local lymph node or adjacent organ metastasis $(n=34)$.

All resection specimens were cut into longitudinal slices 2 to $5 \mathrm{~mm}$ interval. Depths of invasion, lateral and vertical resection margins, and lymphovascular invasions were evaluated. The depth of invasion was classified mucosa and submucosa, and mucosa invasion was subclassified as intraepithelial cancer, cancer invading the lamina propria and invading the muscularis mucosa. The lymphovascular invasion and clearance of the basal and lateral margin were assessed by histopathologic examination. En bloc resection was defined as tumor resection in a single piece. Complete resection was interpreted as all resection margins were tumor free with en bloc resection. ER was conducted by endoscopic mucosal resection (EMR) consist of EMR using a transparent cap procedure (EMR-C), EMR with precutting (EMR-P) and endoscopic submucosal dissection (ESD) with various knives such as hook knife, IT knife-2 or dual knife.

Follow-up examinations were conducted by EGD, chromoendoscopy with lugol solution and biopsy specimen at 3, 6 months and then yearly after ER to examine for local recurrence. CT of chest or PET-CT was performed every year to evaluate the recurrence and metastasis. The primary outcome of ER was determined by type of resection with complete or incomplete resection, histopathologic evaluation with accessing lymphovascular invasion and ER related complication.

\section{Statistical analysis}

Continuous variables are expressed as the mean \pm standard deviation. The chi-square test was used to compare categorical variables, and the t-test was used to compare continuous variables. Survival rates were calculated using Kaplan-Meier methods and the log-rank test was performed to compare survival rate. The p-value of less than 0.05 was considered statistically significant. Statistical analysis was conducted using SPSS version 20.0 (SPSS Inc., Chicago, IL, USA).

\section{RESULTS}

\section{Clinicopathologic features}

Patient's characteristics are summarized in Table 1. ER was conducted with curative intent in all the patients enrolled. Mean age was $66.9 \pm 8.5$ years (range, 45 to 82 years) and the patients were composed of 35 men (94.6\%) and two women (5.4\%) in this study. Mean tumor diameter was $11.5 \pm 5.5 \mathrm{~mm}$ (range, 3 to $31 \mathrm{~mm}$ ) with all histopathologically diagnosed as squamous cell carcinoma. The classification of macroscopic tumor morphology was that type 0-I was five lesion (13.5\%); 0-IIa, four (10.8\%); 0-IIb, nine (24.3\%); 0-IIc, 19 (51.4\%); 0-III, 0 (0\%), respectively. More than half of tumor location were middle esophagus $(n=20$, $54.1 \%)$

\section{Clinical outcomes}

The results of ER are shown in Table 2. Thirty-one lesions (83.8\%) were treated by ESD, four lesions by EMR-P and two lesions by EMR-C. Median duration of ER was 56 minutes (range, 10 to 180 minutes). Overall en bloc resection rate was 34 of 37 (91.9\%). Complete resection rate was 25 of 31 (80.6\%) in ESD group and five of six (83.3\%) in EMR group, and there was no

Table 1. Patient and Tumor Characteristics ( $\mathrm{N}=37)$

\begin{tabular}{|c|c|}
\hline Characteristic & Value \\
\hline \multicolumn{2}{|l|}{ Gender } \\
\hline Male & 35 (94.6) \\
\hline Female & $2(5.4)$ \\
\hline Age, yr & $66.9 \pm 8.5(45-82)$ \\
\hline \multicolumn{2}{|l|}{ Histology } \\
\hline Squamous cell carcinoma & $37(100)$ \\
\hline Others & 0 \\
\hline Tumor size, mm & $11.5 \pm 5.5(3-31)$ \\
\hline \multicolumn{2}{|l|}{ Tumor location } \\
\hline Upper esophagus & $3(8.1)$ \\
\hline Middle esophagus & $20(54.1)$ \\
\hline Lower esophagus & $14(37.8)$ \\
\hline \multicolumn{2}{|l|}{ Dominant macroscopic subtype } \\
\hline 0-I & $5(13.5)$ \\
\hline 0-IIa & $4(10.8)$ \\
\hline $0-\mathrm{IIb}$ & $9(24.3)$ \\
\hline 0-IIc & $19(51.4)$ \\
\hline 0-III & 0 \\
\hline \multicolumn{2}{|l|}{ Pre-existing comorbidity } \\
\hline Cardiovascular disease & $22(59.5)$ \\
\hline Renal dysfunction & $1(2.7)$ \\
\hline Diabetes & $6(16.2)$ \\
\hline Other malignancy & $8(21.6)$ \\
\hline
\end{tabular}

Data are presented as number (\%) or mean \pm SD (range). 
Table 2. Results of Endoscopic Resection and Oncologic Outcomes $(\mathrm{N}=37)$

\begin{tabular}{|c|c|}
\hline Characteristic & Value \\
\hline \multicolumn{2}{|l|}{ Method of endoscopic resection } \\
\hline ESD & $31(83.8)$ \\
\hline EMR-P & $4(10.8)$ \\
\hline EMR-C & $2(5.4)$ \\
\hline \multicolumn{2}{|l|}{ Type of resection } \\
\hline En bloc resection & 34 (91.9) \\
\hline Piecemeal resection & $3(8.1)$ \\
\hline Complete resection rate & $30(81.1)$ \\
\hline \multicolumn{2}{|c|}{ Subclassification of tumor depth } \\
\hline Intraepithelium & $5(13.5)$ \\
\hline Lamina propria & $12(32.4)$ \\
\hline Muscularis mucosa & $10(27.0)$ \\
\hline Submucosa & $10(27.0)$ \\
\hline \multicolumn{2}{|l|}{ Lymphovascular invasion } \\
\hline Positive & $3(8.1)^{*}$ \\
\hline Negative & 34 (91.9) \\
\hline \multicolumn{2}{|l|}{ Lateral resection margin } \\
\hline Positive & $3(8.1)^{*}$ \\
\hline Negative & $34(91.9)$ \\
\hline \multicolumn{2}{|l|}{ Vertical resection margin } \\
\hline Positive & $2(5.4)$ \\
\hline Negative & $35(94.6)$ \\
\hline \multicolumn{2}{|c|}{ Complication of endoscopic resection } \\
\hline Bleeding & 0 \\
\hline Perforation & $3(8.1)$ \\
\hline Stricture & $2(5.4)$ \\
\hline Recurrence & 0 \\
\hline Follow-up duration, mo & $22(4-79)$ \\
\hline
\end{tabular}

Data are presented as number (\%) or median (range).

ESD, endoscopic submucosal dissection; EMR-P, endoscopic mucosal resection with precutting; EMR-C, endoscopic mucosal resection using a transparent cap procedure.

*In one case, both lateral resection margin and lymphovascular invasion were positive.

significant difference $(\mathrm{p}=0.421)$. In one case, two sessions ER was performed for 2 days because of large size and 75\% circumferential extent of tumor in esophagus. The tumor invasion depth was diagnosed as epithelium, five (13.5\%); lamina propria, 12 (32.4\%); muscularis mucosa, 10 (27.0\%); submucosa, $10(27.0 \%)$. The reason of incomplete resection of one patient in EMR group was the presence of high grade dysplasia on lateral margin of resected specimen. In ESD group, two patients were detected the presence of remnant carcinoma on vertical resection margin, two patients on lateral margin and two patients with positive lymphovascular invasion of carcinoma. One patient showed the presence of both lymphovascular invasion and
Table 3. Results of Endoscopic Resection and Additional Treatment Types in Tumor Invasion Depth of the Epithelial and Lamina Propria Group and Muscularis Mucosa and Submucosa Group

\begin{tabular}{lcl}
\hline \multicolumn{1}{c}{ Characteristic } & $\begin{array}{c}\text { Epithelium and } \\
\text { lamina propria } \\
\text { group (n=17) }\end{array}$ & $\begin{array}{c}\text { Muscularis } \\
\text { mucosa and } \\
\text { submucosa } \\
\text { group (n=20) }\end{array}$ \\
\hline $\begin{array}{l}\text { Endoscopic resection results } \\
\text { Depth of tumor invasion }\end{array}$ & $5: 12$ & \\
Epithelium : Lamina propria & & $10: 10$ \\
Muscularis mucosa : Submucosa & $1(5.8)$ & $1(5.0)$ \\
Vertical margin positive & 0 & $3(15.0)^{*}$ \\
Lateral margin positive & 0 & $3(15.0)^{*}$ \\
Lymphovascular invasion positive & & $6(30.0)^{\dagger}$ \\
Types of additional treatment & 0 & 0 \\
Esophagectomy & 0 & $3(15.0)$ \\
Chemotherapy & $1(5.8)$ & $1(5.0)$ \\
Radiotherapy & 0 & $8(40.0)$ \\
Chemoradiotherapy & $13(76.4)$ & $2(10.0)$ \\
No additional treatment & 0 & 0 \\
Transfer to other hospital & $3(17.6)$ & \\
Follow-up loss & &
\end{tabular}

Data are presented as number (\%).

*In one case, both lateral resection margin and lymphovascular invasion were positive; ${ }^{\dagger}$ In one case, esophagectomy followed by adjuvant chemotherapy was performed.

carcinoma in lateral resection margin. In complication, there was no bleeding event, but perforations were developed in three lesions (8.1\%) during ER. After ER, symptomatic esophageal strictures were found in two lesions (5.4\%).

\section{Additional treatment after ER}

Table 3 showed the results of ER and type of additional treatments in the patients of diagnosed tumor invasion depth of epithelium and lamina propria group $(n=17)$ and muscularis mucosa and submucosa group $(\mathrm{n}=20)$. In epithelium and lamina propria group, one patient was received additional treatment consisting of radiotherapy after ER because of vertical margin positive.

In muscularis mucosa and submucosa group, 10 patients were treated ER followed by additional treatment and eight patients did not undergo further additional treatment and two patients were transferred to other hospital for follow-up examination by their demand. Among 10 patients of additional treatments, six patients underwent surgical resection of esophagectomy, three patients with radiotherapy, one patient with chemoradiotherapy. The chemoradiotherapy consisted of two cycles of cisplatin 80 $\mathrm{mg} / \mathrm{m}^{2}$ on days 2 , and continuous infusion of 5-fluorouracil (5FU) $1,000 \mathrm{mg} / \mathrm{m}^{2} /$ day on day 1 to 3 , repeated every 4 weeks with concurrent radiotherapy of 50.4 Gy in 30 fractions. Among six esophagectomy group, the histopathologic assessment of 


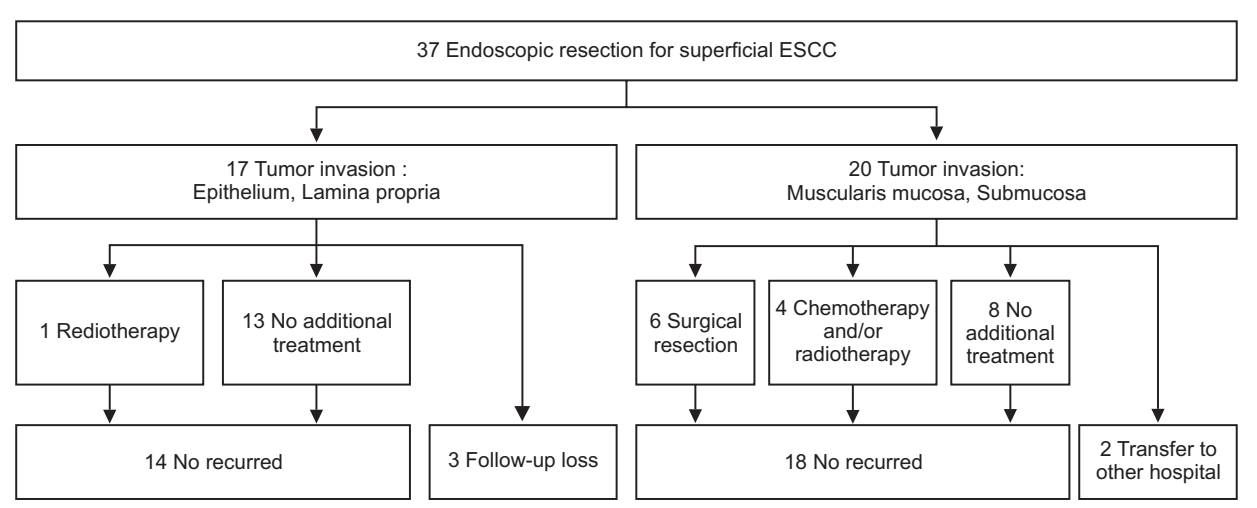

Fig. 1. Clinical course of endoscopic resection patients of superficial esophageal squamous cell carcinoma. ESCC, esophageal squamous cell carcinoma. *In one case, esophagectomy followed by adjuvant chemotherapy was performed. surgically resected specimen showed that in five patients, no residual tumors were detected. In one patient, $1 \mathrm{~mm}$ size of epithelial high grade dysplasia was detected on lateral margin of ER. In regard of lymph node metastasis, two patients (2/6, 33.3\%) were diagnosed as the positive lymph node metastasis in which one patient had a metastatic right recurrent laryngeal lymph node and the other had a metastatic left infra-aortic lymph node. The patient with a metastatic right recurrent laryngeal lymph node received adjuvant chemotherapy composed of six cycles of cisplatin $80 \mathrm{mg} / \mathrm{m}^{2}$ on days 2 , and continuous infusion of 5 -FU $1,000 \mathrm{mg} / \mathrm{m}^{2} /$ day on day 1 to 3 , repeated every 4 weeks.

\section{Survival}

The median follow-up duration was 22 months (range, 4 to 79 months). Synchronous or metachronous lesions of ESCC were not detected in all the patients, and local or metastatic recurrence and death did not occur during follow-up period. Clinical course of patients are represented in Fig. 1.

\section{DISCUSSION}

Esophageal cancer has been treated by surgical resection, which is associated significant mortality that older than 65 remained at 9\%, 30\% higher than any other major cancer operation and postoperative morbidity. ${ }^{11,12}$ The rapid growth of the elderly population, severe concomitant diseases, reduced quality of life after esophagectomy and other primary malignancy make the patients unable to be candidate for the surgical resection. This has led to other approaches to treat such as endoscopic treatment of esophageal cancer, ${ }^{13}$ and several studies had reported the results of ER in ESCC compared with surgical resection. ${ }^{14,15}$ ER has been recognized as the effective treatment for $\mathrm{ESCC}^{14,16}$ and recent prospective study was published evaluating the efficacy and safety of ESD for superficial esophageal neoplasms. ${ }^{17}$

EMR with various methods have been developed such as EMR-P and EMR-C, and disease-specific 5-year survival rate is exceeding $90 \%$ with a low complication rate. ${ }^{18}$ As ER come to be more and more advanced, the new method of ESD has become offered as the standard methods of ER. ESD is superior to EMR as a curative treatment for early ESCC, ${ }^{19,20}$ and the risk of complications such as stenosis and perforation were not significantly different. $^{21}$

ER in our study represents high en bloc resection rate and complete resection rate in superficial ESCC, and is comparable to other studies. The median diameter of lesion in ESD group was $12 \mathrm{~mm}$ (range, 3 to $31 \mathrm{~mm}$ ) and $11 \mathrm{~mm}$ (range, 5 to 18 $\mathrm{mm}$ ) in EMR group ( $\mathrm{p}=0.941)$. EMR was comparable in achieving complete resection in our study because of relatively smaller diameters of tumor by selection bias. Among seven patients of incomplete resection, four patients underwent esophagectomy, one patient received radiotherapy because of severe concomitant disease, one patient refused additional treatment, and one patient was transferred to other hospital.

Regarding ER-related complications, perforations were detected in three patients (8.1\%) during ER. All case of perforation during endoscopic treatment could be treated by complete endoscopic closure with endoclips. And the patients were successfully managed by conservative cares, which included antibiotics and fluid replacement. The strictures were observed in two patients (5.4\%) defined as narrowing that did not allow passage of conventional endoscope in symptomatic patients. All strictures were treated by repeated endoscopic balloon dilation and were managed successfully. No major and serious complications were detected in our study.

ER might be the sufficient treatment option for the lesion as that do not infiltrate beyond the mucosa. In contrast, there is no standard clinical guideline about additional treatment after ER for muscularis mucosal and submucosal invading tumor with a high chance of lymph node metastasis. The additional treatments included esophagectomy, chemotherapy, radiotherapy, and chemoradiotherapy. ${ }^{22}$ We generally recommended esophagectomy as a salvage treatment to patients who were submucosa invasion, incomplete resection of ER and had especially high risk of lymph node metastasis in muscularis mucosa invasion. But, for the reason of significant surgical mortality and morbidity, if patients were unsuitable because of advanced age, severe 
concomitant disease, and patients' desire, alternative treatments were performed for such patients.

In our study, among 10 patients of tumor invasion reaching submucosa, five patients received esophagectomy, three patients by radiotherapy, one patient by chemoradiotherapy respectively. And one patient was transferred to other hospital. This patient was planned to receive esophagectomy due to vertical margin positive, but transferred because of patient's demand. In three patients of radiotherapy, external beam radiation dose was 50.4 Gy in one patients and 54 Gy in two patients. One patient was treated by concurrent chemoradiotherapy without the specific severe complication. The reasons of additional treatment were that five patients were due to invasive tumor depth to submucosa, three patients were positive resection margin, one patient was lymphovascular invasion, and one patient was both. All patients in tumor depth of invasion into submucosa group were followed up without local recurrence or metastasis.

In 10 patients of muscularis mucosa group, one patient underwent surgical resection due to positive lateral margin of resected specimen. This patient had a metastatic right recurrent laryngeal lymph node and received adjuvant chemotherapy. All patients in muscularis mucosa group were followed up without local recurrence or metastasis except one patient being transferred to other hospital. This patient was complete resection of ER and in consultation about additional treatment.

Among 17 patients of epithelium and lamina propria group, only one patient had an additional treatment that was composed of radiotherapy with fractionated 54 Gy by the reason of vertical margin positive. This patient had several severe concomitant diseases and was at advanced age. All patients in epithelium and lamina propria group was maintained contact except three patients who were complete resection state and had become lost during follow-up.

In our study, additional treatments such as esophagectomy, radiotherapy, chemoradiotherapy were offered to the patients with deeper tumor invasion beyond the mucosa. Several studies showed that the metastatic rate was correlated significantly with the depth of tumor invasion. ${ }^{9,23}$ Clinically, the exact diagnosing the depth of tumor invasion before the treatment is still limited with low accuracy. The careful examination of the neoplasm namely macroscopic type of lesion and chromoendoscopy with lugol solution are the most important part of predicting tumor invasion depth and to find out of synchronous lesions. In addition, conventional EUS is necessary to evaluate accurate diagnosis of invasion depth and local lymph node metastasis. Conventional EUS was performed in 34 patients to evaluate the depth of invasion and presence of abnormal or enlarged lymph nodes in our study. The accuracy of T staging for distinguishing between mucosa and submucosa was 58.8\%. Underdiagnosis rate was $26.5 \%$ (nine patients) and overdiagnosis rate $14.7 \%$ (five patients) by EUS. Several studies showed an accuracy in diagnosing submucosal invasion ranges from $33 \%$ to $85 \%{ }^{13,24-26}$
On the basis of our data, it is proposed that before ER, accurate diagnosing the depth of tumor invasion and lymph node metastasis are essential by macroscopic type of lesion and conventional EUS to candidate for ER of superficial ESCC. Because endoscopic view is particularly limited in mucosal and submucosal carcinoma, the histopathologic assessment of endoscopically resected specimens is indispensable and is the principal determinant for decision of additional treatment after ER.

Up to now, esophagectomy with lymph node dissection is the mainstay treatment after ER in incomplete ER or invasive tumor depth because of the risk of local recurrence and lymph node metastasis. But, esophagectomy is associated with the increased mortality, high surgical risk and reduces the quality of life. Patient's age, performance status, and various concomitant diseases should be concerned for offering an appropriate additional treatment. Long-term survival of early esophageal cancer is improving by degrees, thus, quality of life is the decisive factor as well. Radiotherapy and/or chemotherapy are possible to be a valid alternative treatment with similar oncologic outcomes to surgical resection and the effective modalities with less complication than surgical resection. Therefore the choice of the appropriate additional treatment is quite important for patient according to tumor invasion depth of ESCC for this reason. In the current study, the data showed the excellent oncologic outcomes in tumor invasion depth of muscularis mucosa and submucosa regardless of additional treatment in addition to mucosal layer. Accordingly more effective multidisciplinary treatment strategy, the efficacy and usefulness of additional treatment after ER to be supported by well-designed studies is needed certainly. It is difficult to plan, but a randomized controlled study should be performed to evaluate the role of additional treatments.

This study has several limitations such as relatively small size of tumor and sample size, single center study, comparatively short follow-up duration, and retrospective design. Nevertheless, the results of our study represent the first outcomes of endoscopic treatment and proposal of the additional treatment strategy after ER in a group of superficial ESCC with a relating adequate mean follow-up duration of 24.9 months (range, 4 to 79 months) in South Korea. And the data represent the excellent oncologic and clinical results by endoscopic treatment strategy in superficial ESCC. In conclusion, ER with appropriate additional treatment can be considered as an effective and reasonable option, and tailored management after ER can offer an acceptable oncologic outcome in early ESCC.

\section{CONFLICTS OF INTEREST}

The authors have no financial interest or affiliation with any commercial supporter or providers of any commercial services. The authors alone are responsible for the content and writing of this paper. 


\section{REFERENCES}

1. Ferlay J, Shin HR, Bray F, Forman D, Mathers C, Parkin DM. Estimates of worldwide burden of cancer in 2008: GLOBOCAN 2008. Int J Cancer 2010;127:2893-2917.

2. Jung KW, Won YJ, Kong HJ, Oh CM, Seo HG, Lee JS. Cancer statistics in Korea: incidence, mortality, survival and prevalence in 2010. Cancer Res Treat 2013;45:1-14.

3. Pech O, May A, Gossner L, et al. Curative endoscopic therapy in patients with early esophageal squamous-cell carcinoma or highgrade intraepithelial neoplasia. Endoscopy 2007;39:30-35.

4. Oyama T, Tomori A, Hotta K, et al. Endoscopic submucosal dissection of early esophageal cancer. Clin Gastroenterol Hepatol 2005;3:S67-S70.

5. Higuchi K, Tanabe S, Koizumi W, et al. Expansion of the indications for endoscopic mucosal resection in patients with superficial esophageal carcinoma. Endoscopy 2007;39:36-40.

6. Coda S, Lee SY, Gotoda T. Endoscopic mucosal resection and endoscopic submucosal dissection as treatments for early gastrointestinal cancers in Western countries. Gut Liver 2007;1:12-21.

7. Kodama M, Kakegawa T. Treatment of superficial cancer of the esophagus: a summary of responses to a questionnaire on superficial cancer of the esophagus in Japan. Surgery 1998;123:432-439.

8. Nagawa H, Kaizaki S, Seto Y, Tominaga O, Muto T. The relationship of macroscopic shape of superficial esophageal carcinoma to depth of invasion and regional lymph node metastasis. Cancer 1995;75:1061-1064.

9. Takubo K, Makuuchi H, Arima M, Aida J, Arai T, Vieth M. Lymph node metastasis in superficial squamous carcinoma of the esophagus. Pathologe 2013;34:148-154.

10. Japan Esophageal Society. Japanese classification of esophageal cancer, tenth edition: part I. Esophagus 2009;6:1-25.

11. Birkmeyer JD, Stukel TA, Siewers AE, Goodney PP, Wennberg DE, Lucas FL. Surgeon volume and operative mortality in the United States. N Engl J Med 2003;349:2117-2127.

12. Low DE, Bodnar A. Update on clinical impact, documentation, and management of complications associated with esophagectomy. Thorac Surg Clin 2013;23:535-550.

13. Pech 0, May A, Rabenstein T, Ell C. Endoscopic resection of early oesophageal cancer. Gut 2007;56:1625-1634.

14. Shimizu Y, Tsukagoshi H, Fujita M, Hosokawa M, Kato M, Asaka M. Long-term outcome after endoscopic mucosal resection in patients with esophageal squamous cell carcinoma invading the muscularis mucosae or deeper. Gastrointest Endosc 2002;56:387-390.
15. Das A, Singh V, Fleischer DE, Sharma VK. A comparison of endoscopic treatment and surgery in early esophageal cancer: an analysis of surveillance epidemiology and end results data. Am J Gastroenterol 2008;103:1340-1345.

16. Ishihara R, Tanaka H, lishi H, et al. Long-term outcome of esophageal mucosal squamous cell carcinoma without lymphovascular involvement after endoscopic resection. Cancer 2008;112:21662172.

17. Higuchi K, Tanabe S, Azuma M, et al. A phase II study of endoscopic submucosal dissection for superficial esophageal neoplasms (KD0G 0901). Gastrointest Endosc 2013;78:704-710.

18. Pech 0, Gossner L, May A, Vieth M, Stolte M, Ell C. Endoscopic resection of superficial esophageal squamous-cell carcinomas: Western experience. Am J Gastroenterol 2004;99:1226-1232.

19. Shimizu Y, Takahashi M, Yoshida T, et al. Endoscopic resection (endoscopic mucosal resection/endoscopic submucosal dissection) for superficial esophageal squamous cell carcinoma: current status of various techniques. Dig Endosc 2013;25 Suppl 1:13-19.

20. Ishihara $\mathrm{R}$, Iishi H, Uedo $\mathrm{N}$, et al. Comparison of EMR and endoscopic submucosal dissection for en bloc resection of early esophageal cancers in Japan. Gastrointest Endosc 2008;68:1066-1072.

21. Takahashi H, Arimura Y, Masao H, et al. Endoscopic submucosal dissection is superior to conventional endoscopic resection as a curative treatment for early squamous cell carcinoma of the esophagus (with video). Gastrointest Endosc 2010;72:255-264.

22. Kurokawa Y, Muto M, Minashi K, Boku N, Fukuda H; Gastrointestinal Oncology Study Group of Japan Clinical Oncology Group (JCOG). A phase II trial of combined treatment of endoscopic mucosal resection and chemoradiotherapy for clinical stage I esophageal carcinoma: Japan Clinical Oncology Group Study JCOG0508. Jpn J Clin Oncol 2009;39:686-689.

23. Yamashina T, Ishihara R, Nagai K, et al. Long-term outcome and metastatic risk after endoscopic resection of superficial esophageal squamous cell carcinoma. Am J Gastroenterol 2013;108:544-551.

24. May A, Günter E, Roth F, et al. Accuracy of staging in early oesophageal cancer using high resolution endoscopy and high resolution endosonography: a comparative, prospective, and blinded trial. Gut 2004;53:634-640.

25. Zuccaro G Jr, Rice TW, Vargo JJ, et al. Endoscopic ultrasound errors in esophageal cancer. Am J Gastroenterol 2005;100:601-606.

26. Pech 0, May A, Günter E, Gossner L, Ell C. The impact of endoscopic ultrasound and computed tomography on the TNM staging of early cancer in Barrett's esophagus. Am J Gastroenterol 2006;101:2223-2229. 